\title{
Effects of Dibutyl Phthalate on Hepatocytes Histone Acetylation Modification and Liver Function in Rat
}

\author{
Min Li ${ }^{1 \#}$, Qin Lii" ${ }^{2 \#, ~ L i-p i n g ~ Y a n ~}{ }^{3}$, Jia-ling Li ${ }^{1}$, Xu Zhang1 and Ji-yan Cheng1* \\ ${ }^{1}$ Morphology lab, Southwest Medical University, Luzhou 646000, Sichuan, China \\ ${ }^{2}$ Sichuan Vocational College of health and rehabilitation, Zigong 643000, Sichuan, China \\ ${ }^{3}$ Taishan Vocational College of Nursing, Taian 271000, Sandong, China \\ ${ }^{\#}$ Min Li and Qin Li contributed equally to this study
}

*Corresponding author: Ji-yan Cheng, Department of Laboratory Histology and Embryology, Southwest Medical University, Luzhou 646000, Sichuan Province, China

\section{ARTICLE INFO \\ Received: 幽 November 18, 2020 \\ Published: 幽 November 23, 2020}

Citation: Min Li, Qin Li, Li-ping Yan, Jialing Li, Xu Zhang, Ji-yan Cheng. Effects of Dibutyl Phthalate on Hepatocytes Histone Acetylation Modification and Liver Function in Rat. Biomed J Sci \& Tech Res 32(1)-2020. BJSTR. MS.ID.005198.

Keywords: Hepatocytes; Dibutyl Phthalate; Histone Acetyltransferase; Histone Deacetylase

\section{ABSTRACT}

Dibutyl phthalate (DBP) is one of the most commonly used as a plasticizer. Previous studies have shown that DBP is moderately toxic to fish and 40 aquatic invertebrate species, the potential harm of DBP has also attracted human attention [1-3]. DBP can degraded in the liver, but little is known about its mechanism of action in liver cells. Therefore, the purpose of this work was to study the effects of the peroxisome proliferator dibutylphthalate (DBP) on liver function in rat. Histone acetylation and HAT activity regulates gene expression, which is involved in in many human diseases such as neurodegenerative disease and cancer. Therefore in this works, adult male SD rats were used as experimental objects, and DBP was infused into the stomach with corn oil as solvent to explore the residual amount of DBP in the liver and its effect on the morphological structure of rat hepatocytes, the expression of HAT-PCAF, HDAC-1were examined to evaluate nuclear histone acetylation level and total histone acetyltransferase (HAT) and deacetylase (HDAC) activity in the effects of the DBP on liver function in rat.

Conclusion: DBP remained at a high level in the rat liver for a long time. The mechanism may be through the influence of histone acetylation modification pathway of hepatocytes and metabolism of organic substances in hepatocytes, leading to changes in the structure and function of hepatocyte nucleus, nucleosome and organelle, and finally damage the liver function.

\section{Introduction}

Dibutyl phthalate (DBP) is one of the most commonly used as additives for polyvinyl chloride (Polyvinyl chloride polymer, PVC). With the extensive use of PVC in medical supplies, cosmetics, food packaging and other fields, the potential harm of DBP has also attracted human attention [1-3]. DBP is water-soluble and can be easily transferred into monobutyl phthalate (MBP) and its glucuronide extensively in vivo. DBP is degraded in the liver and then excreted through the kidney after entering the body. Previous reports have indicated that intake of DBP will damage the structure and function of the liver and kidney [4]. DBP is moderately toxic to fish and 40 aquatic invertebrate species. Recently, the potential harm of DBP has also attracted human attention [1-3]. At present, it is believed that the effect of DBP impact on human health is mainly due to its interference with the synthesis, secretion, transport, binding, active response, metabolism, degradation and so on of the organism's own hormones. Histone acetylation is conducive to the dissociation of DNA and histone octamer, so that various transcription factors can specifically bind to DNA binding sites and activate gene transcription. On the other hand, the deacetylation of histone has the opposite effect. Histone acetyltransferase (HAT) promotes histone acetylation and increases gene expression, histone deacetylase (HDAC) makes chromatin densely curled, and gene transcription is inhibited [5]. HAT-PCAF belonging to the GNAT (Gcn5-related N-acetyltransferases) family of HAT. It is derived 
from human and mouse and can specifically bind to H3/H4 histone in vivo. Histone acetylation and HAT activity are involved in in many human diseases such as neurodegenerative disease and cancer. In this study, adult male SD rats were used as experimental objects, and DBP was infused into the stomach with corn oil as solvent to explore the residual amount of DBP in the liver and its effect on the morphological structure of rat hepatocytes, the expression of HATPCAF, HDAC-1 were examined to reflect liver function.

\section{Materials and Methods}

\section{Reagents and Apparatus}

DBP was purchased from Qiaoke Chemical Co., Ltd (Dongguan, China). Immunohistochemical staining kit were obtained from Bioss Biotechnology Co., Ltd (Beijing, China). Antibodies against HAT-PCAF and HDAC-1 were obtained from Cell Signal. HRPconjugated secondary antibodies was purchased from Abcam (Cambridge, MA, USA). Quantum dot labeling kit was purchased from Jiayuan Quantum Dots Co., Ltd.( Wuhan, China). Fluorescence microscope (Olympus, Japan). Optical microscope (Leica, Germany). Transmission electron microscopy JEOL-1400 (JEOL, Japan).

\section{Animal Experiment}

80 healthy adult male Sprague-Dawley (SD) rats, weighing $246 \pm 17 \mathrm{~g}$, were purchased from the Animal Experimental Center of Southwest Medical University. Water and food were provided ad libitum. The rats were randomly divided into four groups. Each group was given DBP $0 \mathrm{mg} / \mathrm{kg}$ (control group), $50 \mathrm{mg} / \mathrm{kg}$ (low dose group), $250 \mathrm{mg} / \mathrm{kg}$ (middle dose group) and $500 \mathrm{mg} / \mathrm{kg}$ (high dose group) every day. DBP was intragastrically administered with corn oil as a solvent, and the dose was $1 \mathrm{ml}$ per $100 \mathrm{~g}$ body weight for 21 days. On the 24th day after anesthesia, the liver tissues were taken for follow-up experiments and quickly stored in liquid nitrogen. In the course of animal experiment, it complies with the ethical standards formulated by the experimental animal ethics committee.

\section{High Performance Liquid Chromatograph (HPLC)}

The control sample was accurately weighed, dissolved in isopropanol and made to a constant volume to make a control solution with a concentration of $0.1677 \mathrm{mg} / \mathrm{ml}$. Precisely weigh $1.00 \mathrm{~g}$ of the sample, the tissue was comminuted in $3 \mathrm{ml}$ isopropanol and the supernatant was used to analyze after centrifugation. The peak area was used to calculate the residuary DBP with HPLC. Detection conditions: chromatographic; column: Dima Kromasil C18 column $(250 \mathrm{~mm} \times 4.6 \mathrm{~mm}, 5 \mu \mathrm{m})$; mobile phase: acetonitrile-water (85:15); detection wavelength: $225 \mathrm{~nm}$; column temperature: $30^{\circ} \mathrm{C}$; flow rate: $1.0 \mathrm{ml} / \mathrm{min}$; injection volume: $10 \mu \mathrm{l}$ [6].

\section{Morphological Analysis}

Hematoxylin-eosin (HE) staining: $1 \mathrm{~cm}^{3}$ of liver tissue fixed with $4 \%$ formaldehyde, dehydrated with gradient ethanol, rendered transparent by chloroform, dipped in wax and embedded, and made into paraffin sections with a thickness of $5 \mu \mathrm{m}$. The slices were dewaxed with xylene, dehydrated with gradient ethanol, stained with hematoxylin, decolorized with hydrochloric ethanol, returned to blue with ammonia, stained with eosin, dehydrated with gradient ethanol, rendered transparent by xylene, and sealed with resin [7]. Transmission electron microscopy (TEM): Five pieces of liver tissue with a volume of about $1 \mathrm{~mm}^{3}$ immersed in $2.5 \%$ glutaraldehyde were taken. The samples were fixed with glutaraldehyde at $4^{\circ} \mathrm{C}$ for $2 \mathrm{~h}$, washed with PBS, fixed with $1 \%$ osmic acid at $4^{\circ} \mathrm{C}$ for 3 hours, washed with distilled water, dehydrated with gradient acetone then embedded in epoxy resin. The sample was sectioned to $60 \mathrm{~nm}$ and stained by uranium lead double staining [8].

\section{Immunohistochemistry}

The slices were dewaxed with xylene, dehydrated with gradient ethanol, and antigen was repaired by sodium citrate thermal repair method for 15 min. After cooling, the slices washed with PBS, and endogenous peroxidase was inactivated with $3 \% \mathrm{H}_{2} \mathrm{O}_{2}$. The sections were blocked with goat serum, incubated overnight at $4{ }^{\circ} \mathrm{C}$ with $1: 100$ diluted HAT-PCAF and HDAC-1 primary antibody, washed with PBS, incubated with second antibody at room temperature for $30 \mathrm{~min}$, washed with PBS, and colored by DAB. Distilled water under the microscope to stop color development, dehydrated with gradient ethanol, rendered transparent by xylene, and sealed with resin [9].

\section{Immunofluorescence}

The slices were dewaxed with xylene, dehydrated with gradient ethanol, washed with PBS, inactivated by $3 \% \mathrm{H} 2 \mathrm{O} 2$, sealed with goat serum, and incubated overnight at $4{ }^{\circ} \mathrm{C}$ with $1: 100$ diluted HAT-PCAF and HDAC-1 primary antibody, washed with PBS, incubated with quantum dots labeled second antibody complex at room temperature for $30 \mathrm{~min}$, washed with PBS, and sealed with glycerol [10].

\section{Serum Enzyme Analysis}

Blood from cardiac cavity of rats was taken to collected serum, and the alanine aminotransferase (ALT), aspartate aminotransferase (AST) and alkaline phosphatase (ALP) were determined by Hitach-7060 automatic biochemical analyzer [11].

\section{Statistics}

SPSS 13.0 software and rank sum test were used for statistical analysis. All data were expressed as mean \pm standard deviation, $\mathrm{P}$ $<0.05$ showed that the difference was statistically significant [12].

\section{Result}

\section{Determination of Residual DBP In the Rat Liver}

The residue of DBP in rat liver was detected by HPLC. The results showed that the content of DBP in the liver was significantly different between the control group and each exposed group Figure 1. Quantitative Analysis reflected that the content of DBP 
in the control group was very low. As the dose was increased, the residual amount of DBP in the liver was gradually raised, reaching the highest in the high dose group. The difference between each exposure groups compared with the control was statistically significant $(\mathrm{p}<0.05)$ (Table 1).
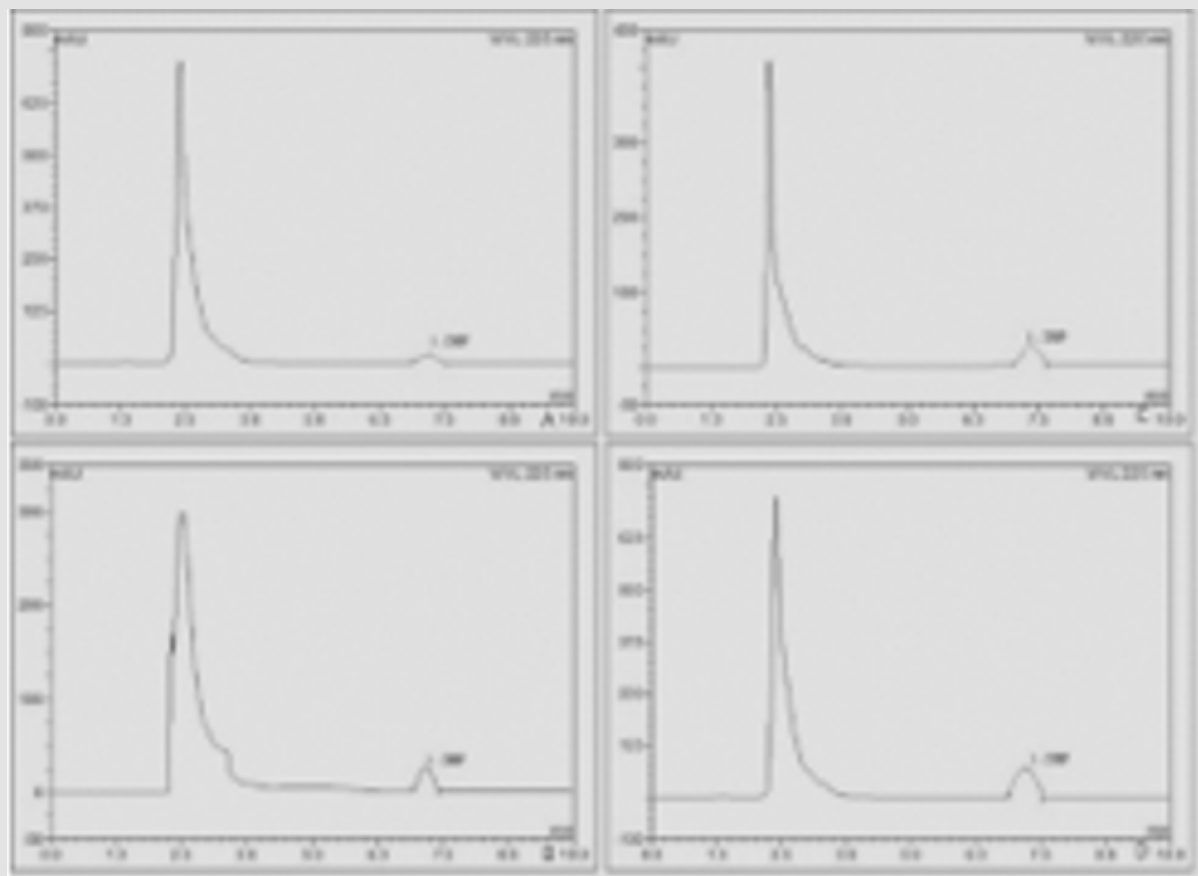
A. Control group
B. Low dose Group
C. Medium dose group
D. High dose group

Figure 1: Detection of DBP residue in liver. Each of the four groups of rats was given DBP 0, 50, 250, and $500 \mathrm{mg} / \mathrm{kg}$ each day. After continuous gavage for 21 days, the samples were taken and the DBP content in liver tissue was detected by HPLC.

Table 1: Determination of DBP content in rat liver by HPLC.

\begin{tabular}{|c|c|c|}
\hline Groups & $\mathbf{n}$ & DBP content $(\boldsymbol{\mu g} / \mathbf{g})$ \\
\hline control group & 20 & $0.0917 \pm 0.0015$ \\
\hline low dose group & 20 & $0.2819 \pm 0.0055^{*}$ \\
\hline middle dose group & 20 & $0.4839 \pm 0.0083 \triangle$ \\
\hline high dose group & 20 & $0.9189 \pm 0.0062 \boldsymbol{\triangle}$ \\
\hline
\end{tabular}

Quantitative analysis of the results obtained in the HPLC to calculate the content of DBP in the liver of each group, the value is expressed as $x^{-} \pm S D, n=20$. Note: *Compared with the control group $\mathrm{P}<0.05 ; \triangle$ Compared with the control group $\mathrm{P}<0.05$; $\Delta$ Compared with the control group $\mathrm{P}<0.05$.

\section{Morphological Changes of Rat Liver Tissue After DBP Gavage}

HE staining of liver tissue showed that in the control group, the arrangement of hepatocytes was regular, the morphological structure was normal, the structure of nucleus was clear, and the structure of hepatic sinusoid was normal Figure 2. However, the arrangement of cells in each exposed group was gradually irregular, and the vacuole structure could be seen in the cytoplasm. With the increase of the DBP dose, the number of cells with vacuoles in the cytoplasm increased significantly. In the high dose group, the arrangement of cells were very irregular, the cells were vacuoleslike, the hepatic sinusoid dilated and obvious congestion appeared Figure 2. The ultrastructure of hepatocytes was observed by TEM, the results showed that in the control group, the arrangement of hepatocytes was regular, the cell morphology was more regular, the nuclear structure was clear, the nucleolus was obvious, and the cytoplasm was abundant. A large number of organelles such as mitochondria and endoplasmic reticulum can be seen, and the morphological structure is normal Figure 3. In the exposed group, the cell morphology was irregular, the nuclear structure was irregular, several obvious changes could be seen in the organelles and cytoplasm: a large number of lipid droplets appeared and a large number of coarse lipid droplets appeared with the increase of exposure dose, and the mitochondria showed slight pyknosis and increased electron density, and there is a large amount of glycogen Figure 3. 

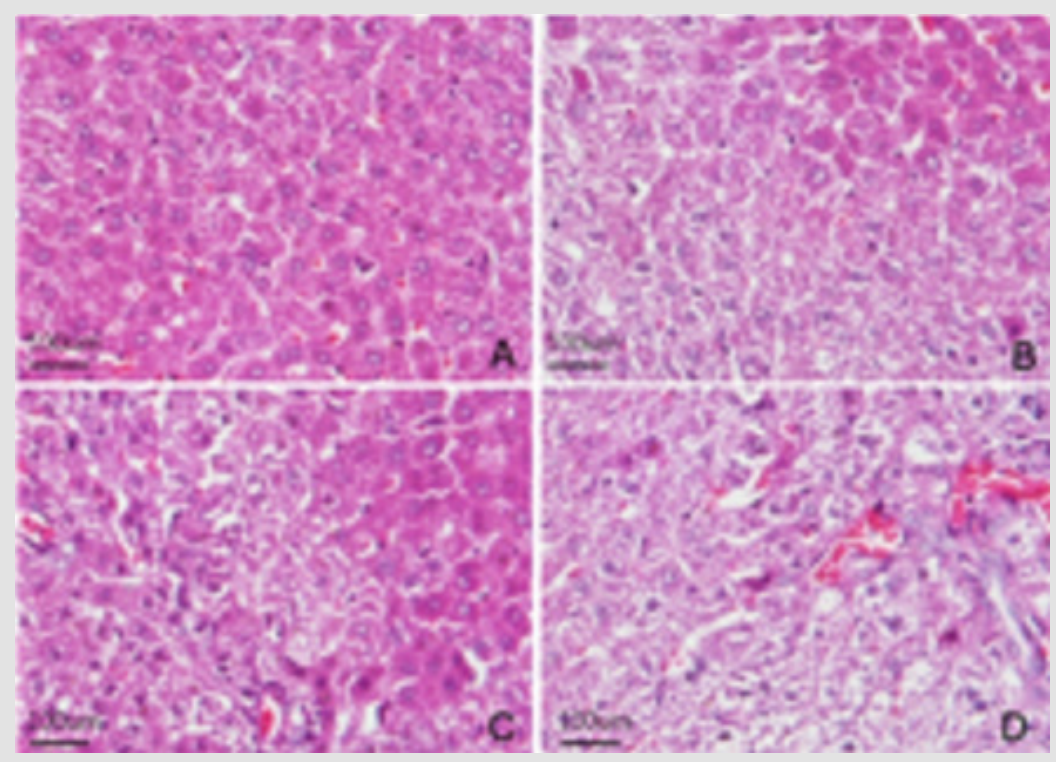
A. Control group
B. Low dose Group
C. Medium dose group
D. High dose group. Bar $=100 \mu \mathrm{m}$.

Figure 2: Morphological effects of DBP on liver tissue of rats in each exposed group. Liver tissues were taken from four groups of rats fed for 21 days to make paraffin sections, and then HE staining was performed to observe the morphological changes of liver tissues of rats in each group.

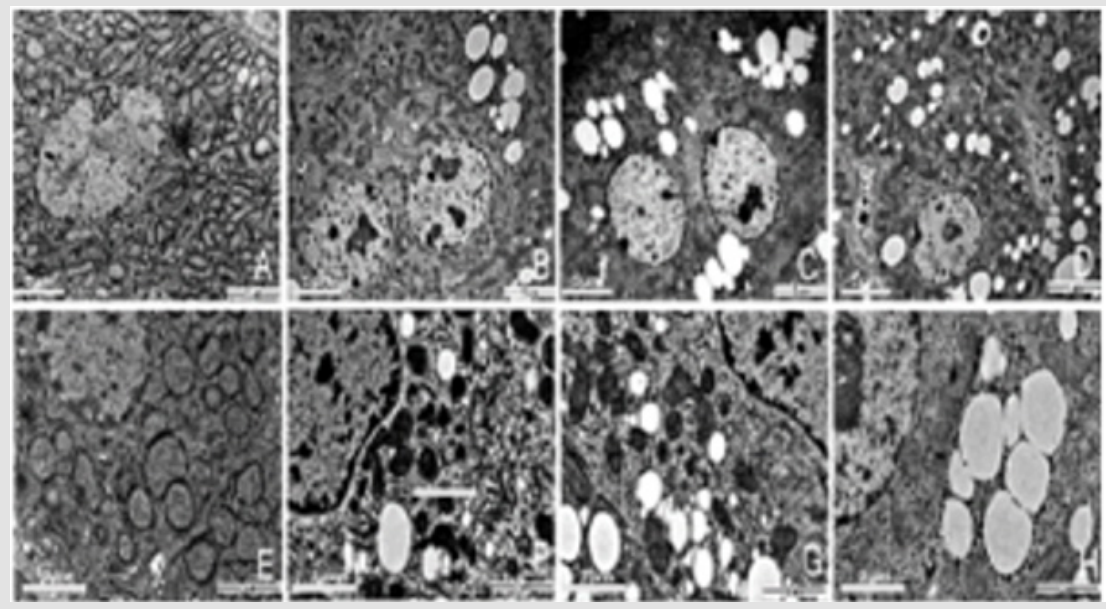
A. Control group
B. Low dose Group
C. Medium dose group
D. High dose group. Observation condition is 4000 times magnification, Bar $=5 \mu \mathrm{m}$.
E. Control group
F. Low dose Group
G. Medium dose group
H. High dose group. Observation condition is 12000 times magnification, Bar $=2 \mu \mathrm{m}$.

Figure 3: TEM observation of the effects of DBP on the ultrastructure of rat hepatocytes. The four groups were observed by TEM to observe the ultrastructural changes of liver cells in each dose group. 
HAT-PCAF of hepatocytes increased with the increase of DBP dose

To examine whether TA modulates HAT activity, we initially measured HAT activity in a cell-free system. The results of hepatocytes HAT-PCAF and HDAC-1 immunohistochemical staining showed that HDAC-1 and HAT-PCAF were mainly expressed in cytoplasm. HDAC-1 expressed weak in control group, HAT-PCAF expression gradually increased with the increased of the dose, HAT-PCAF absorbance and PN-area in the exposed group were significantly different from the control group $(\mathrm{P}<0.05)$ Figure 4;
The expression of HDAC-1 was not significantly different from the control group $(\mathrm{P}>0.05)$ Figure 4 (Table 2). Hepatocytes quantum dot labeling HAT-PCAF、HDAC-1 staining results showed that, HAT-PCAF and HDAC-1 after labeling stimulated the red fluorescence, mainly expressed in the cytoplasm Figure 5; In the exposed group, the expression of HAT-PCAF increased with the increase of the exposure dose, and the fluorescent intensity of HATPCAF was significant different from the control group $(\mathrm{P}<0.05)$; HDAC1 expression strength in each group, there was no significant difference Figure 5, (Table 3).
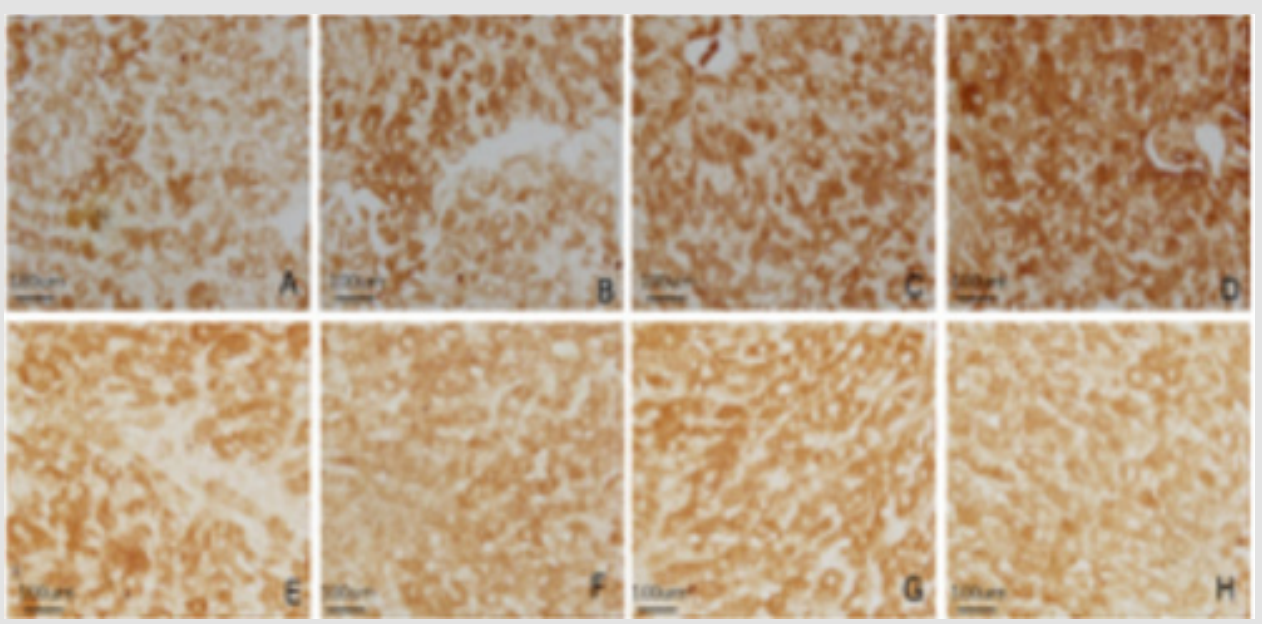
A. Control group HAT-PCAF
B. Low dose group HAT-PCAF
C. Middle dose group HAT-PCAF
D. High dose group HAT-PCAF
E. Control group HDAC-1
F. Low dose group HDAC- 1
G. Middle dose group HDAC-1
H. High dose group HDAC-1, Bar=100 $\mu \mathrm{m}$.

Figure 4: The effect of DBP on histone acetylation of liver cells. Immunohistochemical staining was performed on HAT-PCAF and HDAC-1 in rat hepatocytes.

Table 2: DImmunohistochemical absorbance and positive reaction area of each group.

\begin{tabular}{|c|c|c|c|c|c|}
\hline \multirow{2}{*}{ Groups } & \multirow{2}{*}{$\mathbf{n}$} & \multicolumn{2}{|c|}{ HAT } & \multicolumn{2}{|c|}{ HDAC-1 } \\
\cline { 2 - 5 } & Absorbance & Pn-Area & $0.207 \pm 0.026$ & $36.25 \pm 5.16$ \\
\hline control group & 20 & $0.156 \pm 0.015$ & $27.26 \pm 3.21$ & $0.196 \pm 0.032 \nabla$ & $36.47 \pm 2.55 \nabla$ \\
\hline middle dose group & 20 & $0.261 \pm 0.023^{*}$ & $37.41 \pm 4.18^{*}$ & $0.215 \pm 0.041 \nabla$ & $38.21 \pm 4.87 \nabla$ \\
\hline high dose group & 20 & $0.272 \pm 0.039 \triangle$ & $41.59 \pm 5.28 \triangle$ & $0.182 \pm 0.053 \nabla$ & $35.76 \pm 3.21 \nabla$ \\
\hline
\end{tabular}

Theimmunohistochemical staining results of livercells HAT-PCAF and HDAC-1 ineach group were analyzed for immunohistochemical absorbance and positive reaction area. Note: ${ }^{*} \mathrm{P}<0.05$ compared with the control group; $\triangle \mathrm{P}<0.05$ compared with the control group; $\Delta \mathrm{P}<0.05$ compared with the control group; $\nabla \mathrm{P}>0.05$ compared with the control group, the value is expressed by $x^{-} \pm S D, n=20$. 

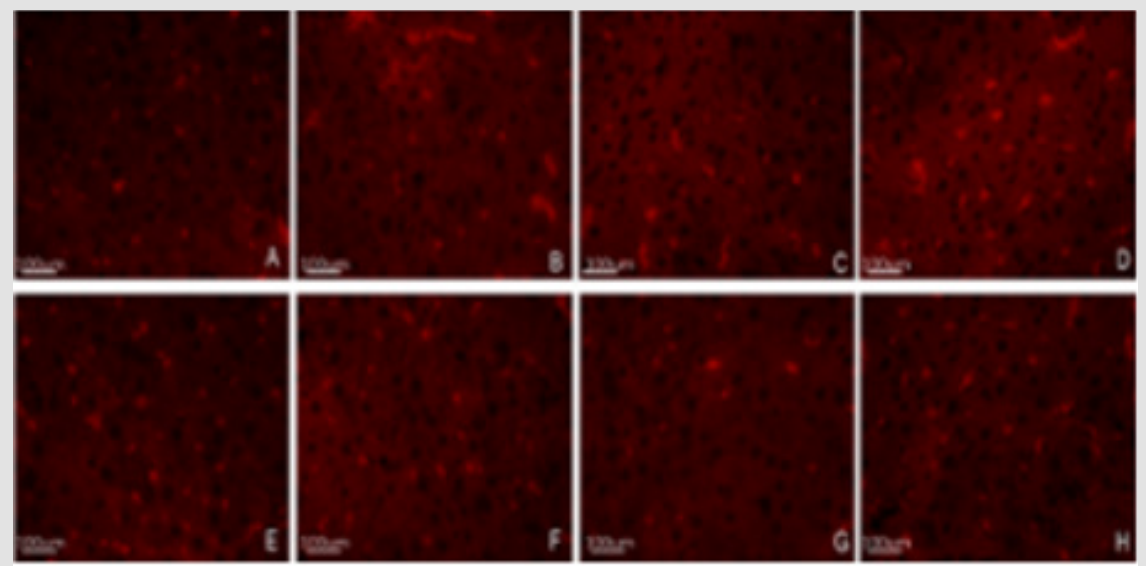
A. Control group HAT-PCAF
B. Low dose group HAT-PCAF
C. Middle dose group HAT-PCAF
D. High dose group HAT-PCAF
E. Control group HDAC-1
F. Low dose group HDAC-1
G. Middle dose group HDAC-1
H. High dose group HDAC-1, Bar=100 $\mu \mathrm{m}$.

Figure 5: Immunofluorescence detection of liver cells HAT-PCAF and HDAC-1. To further examine the effect of DBP on the histone acetylation of hepatocytes, four groups of rats were selected for immunofluorescence detection with quantum dot labeling.

Table 3: Quantitative analysis of the immunofluorescence results of each group.

\begin{tabular}{|c|c|c|c|}
\hline Groups & n & HAT & HDAC-1 \\
\hline control group & 20 & $36.21 \pm 5.98$ & $34.56 \pm 4.39$ \\
\hline low dose group & 20 & $38.09 . \pm 8.14^{*}$ & $32.72 \pm 2.94 \nabla$ \\
\hline middle dose group & 20 & $41.26 \pm 4.26 \triangle$ & $35.48 \pm 2.86 \nabla$ \\
\hline high dose group & 20 & $49.50 \pm 5.91 \boldsymbol{\Delta}$ & $37.25 \pm 5.71 \nabla$ \\
\hline
\end{tabular}

Note: ${ }^{*} \mathrm{P}<0.05$ compared with the control group; $\triangle \mathrm{P}<0.05$ compared with the control group; $\boldsymbol{\Delta} \mathrm{P}<0.05$ compared with the control group; $\nabla \mathrm{P}>0.05$ compared with the control group, the value is expressed by $x^{-} \pm S D, n=20$.

\section{Effect of DBP On Liver Function in Rats}

To reflect the liver functionin the experiment, we examined the aspartate transaminase (AST), alkaline phosphatase (ALP) and alanine transaminase (ALT) expression in liver blood in each group , and the results showed that the level of AST, ALP and ALT were significantly increased in the exposed group, which indicated that DBP significantly damaged the function of rat hepatocytes. The possible mechanism is that DBP remains in high concentrations in hepatocytes, interferes with the activity of enzymes related to histone acetylation modification, affects histone modification, and destroys the nucleosome, nucleus and organelle structure of hepatocytes. Each group rat's blood liver function analysis results showed that ALT, AST and ALP obviously increased with the increased dose, there was a significant difference compared with the control group $(\mathrm{P}<0.05)$ (Table 4). The four groups were observed by TEM to observe the ultrastructural changes of liver cells in each dose group. A, Control group; B, Low dose Group; C, Medium dose group; D, High dose group. Observation condition is 4000 times magnification, Bar $=5 \mu \mathrm{m}$. E, Control group; F, Low dose Group; G, Medium dose group; H, High dose group. Observation condition is 12000 times magnification, Bar $=2 \mu \mathrm{m}$. HAT-PCAF; B, Low dose group HAT-PCAF; C, Middle dose group HAT-PCAF; D, High dose group HAT-PCAF; E, Control group HDAC-1; F, Low dose group HDAC- 1; G, Middle dose group HDAC-1; H, High dose group HDAC -1 , Bar $=100 \mu \mathrm{m}$.

Table 4: Effect of DBP on liver function in rats. Four groups of rats were gavaged for 21 days, and the blood was collected from the heart cavity of rats to determine the serum ALT, AST and ALP content. Note: ${ }^{*} \mathrm{P}<0.05$ compared with the control group; $\triangle \mathrm{P}<0.05$ compared with the control group; $\Delta \mathrm{P}<0.05$ compared with the control group.

\begin{tabular}{|c|c|c|c|c|}
\hline Groups & $\mathbf{n}$ & ALT & AST & ALP \\
\hline control group & 20 & $28.12 \pm 2.56$ & $17.8 \pm 3.61$ & $62.2 \pm 8.16$ \\
\hline $\begin{array}{c}\text { low dose } \\
\text { group }\end{array}$ & 20 & $78.26 \pm 5.41^{*}$ & $175.1 \pm 11.44^{*}$ & $175.6 \pm 17.28^{*}$ \\
\hline $\begin{array}{c}\text { middle dose } \\
\text { group }\end{array}$ & 20 & $75.92 \pm 7.16 \Delta$ & $191.3 \pm 12.79 \Delta$ & $219.24 \pm 21.19 \Delta$ \\
\hline $\begin{array}{c}\text { high dose } \\
\text { group }\end{array}$ & 20 & $77.86 \pm 9.73 \triangle$ & $152.7 \pm 15.53 \triangle$ & $224.9 \pm 21.28 \triangle$ \\
\hline
\end{tabular}

To further examine the effect of DBP on the histone acetylation of hepatocytes, four groups of rats were selected for 
immunofluorescence detection with quantum dot labeling. A, Control group HAT-PCAF; B, Low dose group HAT-PCAF; C, Middle dose group HAT-PCAF; D, High dose group HAT-PCAF; E, Control group HDAC-1; F, Low dose group HDAC-1; G, Middle dose group HDAC-1; H, High dose group HDAC-1, Bar $=100 \mu \mathrm{m}$.

\section{Discussion}

As an important plasticizer, DBP is widely used in many fields. The effect of DBP on human health has been recognized, mainly affecting reproductive function and neurological function. Its action mechanism is mainly similar to hormones, interfering with cell metabolism, proliferation, differentiation and so on $[13,14]$. Histone is a very important component of nuclear proteins and participates in the formation of nucleosomes. Histone ends can be modified by acetylation, methylation, phosphorylation, poly-ADP ribose acylation, ubiquitin and other modifications, thus affecting the gene expression, structure and function of cells. The study on the relationship between histone modification and chromatin structure changes and gene activity inhibition is a very important research topic. Histone acetylation is regulated by HAT and HDAC. The main purpose of this study was to explore the effects of DBP on the structure and function of hepatocytes in rats, and to detect the residual amount of DBP, the expression of histone acetylation and deacetylation related enzymes HAT-PCAF and HDAC-1, and the changes of liver function by various techniques, so as to explore the possible toxic mechanism of DBP on rat hepatocytes.

According to the WHO standard, the body's tolerable daily intake of plasticizer (Tolerable Daily Intake, TDI) is $0.025 \mathrm{mg} /$ $\mathrm{kg}[15,16]$. For adults weight $60 \mathrm{~kg}$, the tolerable daily intake of plasticizer is $1.5 \mathrm{mg}$. In this study, the results of HPLC showed that the residual amount of DBP in the liver of rats exposed to DBP was much higher than this value, and the highest in the high dose group, up to $0.9189 \mu \mathrm{g} / \mathrm{g}$. Thus, it can be seen that DBP remains in high concentration in rat liver and hepatocytes, so it is bound to damage the structure and function of hepatocytes. The mechanism may be that DBP is degraded in hepatocytes, and the degradation rate is lower than the uptake rate. In this study, immunohistochemical staining and immunofluorescence results showed that oral intake of DBP was increased the expression of HAT-PCAF in liver, but had no significant effect on the expression of HDAC-1. These results suggest that the effect of DBP on rat liver and hepatocytes may be that it breaks the steady state of histone acetylation by changing the activity of histone acetylating enzyme in hepatocytes, especially by promoting the expression of HAT-PCAF and histone acetylation, thus changing the structure and function of nucleus and gene expression, and directly interfering with the metabolism of sugar, protein and lipid in hepatocytes.

Damage the biotransformation, protein synthesis and other functions of the liver, and even lead to irreversible destruction [17]. HE staining and TEM showed that in the exposed group, the arrangement of hepatocytes was irregular, lipid droplets accumulated in the cytoplasm, the morphology and structure of hepatocytes and their organelles were damaged. The damage mechanism may be that DBP changes the morphology and structure of nucleus, nucleosome, cell and abnormal gene expression by changing histone modification. From the aspects of microstructure and ultrastructure, it shows the structural damage of nucleus, nucleosome, organelle and the disorder of organic matter metabolism [18]. ALT is the main index for the diagnosis of hepatocyte parenchyma damage, and its level is parallel to the severity of liver injury. The significance of elevated AST is similar to that of ALT in the diagnosis of hepatitis. ALP is excreted through the biliary tract, so the excretion dysfunction of liver diseases, biliary diseases and other diseases can increase ALP [19]. In the experiment, the results of blood liver function analysis of rats in each group showed that ALT, AST and ALP were significantly increased in the exposed group, which indicated that DBP significantly damaged the function of rat hepatocytes. The possible mechanism is that DBP remains in high concentrations in hepatocytes, interferes with the activity of enzymes related to histone acetylation modification, affects histone modification, and destroys the nucleosome, nucleus and organelle structure of hepatocytes. It changed the metabolism of organic matter in hepatocytes, resulting in the increase of ALT, AST and ALP in the liver, thus damaging the liver function [20].

\section{References}

1. Daniel J W (1978) Toxicity and Metabolism of Phthalate Esters. Clinical Toxicology 13(2): 257-268.

2. Seth Prahlad K (1982) Hepatic Effects of Phthalate Esters. Environmental Health Perspectives 45(NOV): 27-34.

3. Fan Guangtao, Xie Jingchao, Yoshino Hiroshi, U.Yanagi, Kenichi Hasegawa, et al. (2017) Environmental conditions in homes with healthy and unhealthy schoolchildren in Beijing, China. Building \& Environment 112(FEB.): 270-284.

4. Kim Johanna Inhyang, Hong Yun Chul, Shin Choong Ho, Young AhLee, Youn-HeeLim, et al. (2017) The effects of maternal and children phthalate exposure on the neurocognitive function of 6-year-old children. Environmental Research 156(JUL.): 519-525.

5. Qiao Yunbo, Wang Ran, Yang Xianfa (2014) Dual Roles of Histone H3 Lysine 9 Acetylation in Human Embryonic Stem Cell Pluripotency and Neural Differentiation. Journal of Biological Chemistry 290(16): 25082520 .

6. Wen Qiongfang, Zhang Yanfang, Zhang Jingqing (2016) Simultaneous determination of 6 organic acids, 3 nucleosides, and ephedrine in Pinellia ternata by HPLC.

7. Donde Hridgandh, Ghare Smita, Joshi-Barve Swati, JingWenZhang, Manicka V Vadhanam, et al. (2019) Tributyrin Inhibits Ethanol-Induced Epigenetic Repression of CPT-1A and Attenuates Hepatic Steatosis and Injury. Cellular and molecular gastroenterology and hepatology,2019) 9(4): 569-585.

8. Jiong $\mathrm{Hu}$, Sarah Dziumbla, Jihong Lin, Sofia-Iris Bibli, Sven Zukunft, et al. (2017) Inhibition of soluble epoxide hydrolase prevents diabetic retinopathy. Nature 552(7684): 248-252.

9. Wang Zhichao, Cao Dan, Li Chonghui, Lihua Min, Gang Wang, et al.(2019) Mediator MED23 regulates inflammatory responses and liver fibrosis. PLoS Biology 17(12): 
10. XiaoPing Qi, YunYe, ZhongShi Lin, Liang Xu, Hao Yu, et al. (2016) Dual-Quantum-Dots-Labeled Lateral Flow Strip Rapidly Quantifies Procalcitonin and C-reactive Protein. Nanoscale Research Letters,2016): 167 (2016).

11. Zhou Jiyu, Huang Ningning, Guo Yitong (2018) Combined obeticholic acid and apoptosis inhibitor treatment alleviates liver fibrosis. Acta Pharmaceutica Sinica B 9(3): 526-536.

12. Zahid Kashif Rafiq, Khan Abdur Rehman Raza, Yao Shun, Umar Raza, Deming Gou, et al.(2019) mTOR/HDAC1 crosstalk mediated suppression of ADH1A and ALDH2 links alcohol metabolism to hepatocellular carcinoma onset and progression in silico. Frontiers in oncology 9: 1000.

13. Li Tiancui, Fan Yaocheng, Cun Deshou, XiaoyongSongabYanranDai, et al. (2019) Treatment performance and microbial response to dibutyl phthalate contaminated wastewater in vertical flow constructed wetland mesocosms. Chemosphere,2019) 246: 125635.

14. Struhl Kevin (1998) Histone acetylation and transcriptional regulatory mechanisms. Genes \& development 12(5): 599-606.

15. Turco Vincenzo Lo, Di Bella Giuseppa, Potortì Angela Giorgia (2015) Determination of plasticizer residues in tea by solid phase extractiongas chromatography-mass spectrometry. European Food Research and Technology 240(2): 451-458.

\section{ISSN: 2574-1241}

DOI: $10.26717 /$ BJSTR.2020.32.005198

Ji-yan Cheng. Biomed J Sci \& Tech Res

(C) (i) This work is licensed under Creative

Submission Link: https://biomedres.us/submit-manuscript.php
16. Chan Kang Se, Mu Lee Byung (2005) DNA methylation of estrogen receptor $\alpha$ gene by phthalates. Journal of Toxicology and Environmental Health, Part A 68(23-24): 1995-2003.

17. Kelly RDW, Chandru Aditya, Watson PJ, Y. Song, M Blade, et al. (2018) Histone deacetylase (HDAC) 1 and 2 complexes regulate both histone acetylation and crotonylation in vivo. Scientific reports 8(1): 1-10.

18. Li Xiaohui, Yin Pinghe, Zhao Ling (2017) Effects of individual and combined toxicity of bisphenol A, dibutyl phthalate and cadmium on oxidative stress and genotoxicity in HepG 2 cells. Food and chemical toxicology 105: 73-81.

19. Yang Zhengtao, Liu Xiao, Wang Yanan, Shuangqiu Li, Chaoqun Wang,et al. (2019) Magnolol prevents acute alcoholic liver damage by activating PI3K/Nrf2/PPAR $\gamma$ and inhibiting NLRP3 signaling pathway. Frontiers in Pharmacology 10: 1459.

20. Jiao Yaqi, Tao Yue, Yang Yang, Tuyiringire Diogene,Hui Yu, et al. (2019) Monobutyl phthalate (MBP) can dysregulate the antioxidant system and induce apoptosis of zebrafish liver. Environmental Pollution): 113517.

$\begin{array}{ll}\text { BIOMEDICAL } & \text { Assets of Publishing with us } \\ \text { RESEARCHES } & \text { - Global archiving of articles } \\ \text { - Immediate, unrestricted online access } & \text { - Rigorous Peer Review Process } \\ & \text { - Authors Retain Copyrights } \\ & \end{array}$

